DOI: 10.1002/adem.((please add manuscript number))

\title{
Annealing-induced Hardening in Ultrafine-grained Ni-Mo Alloys **
}

By Jenö Gubicza*, Pedro Henrique R. Pereira, Garima Kapoor, Yi Huang, V. Subramanya Sarma, and Terence G. Langdon

[*] Prof. Dr. Jenő Gubicza, Ms. Garima Kapoor

Department of Materials Physics, Eötvös Loránd University, P.O.B.32, Budapest, H-1518, Hungary

E-mail: jeno.gubicza@ttk.elte.hu

Dr. Pedro Henrique R. Pereira, Dr. Yi Huang, Prof. Dr. Terence G. Langdon Materials Research Group, Faculty of Engineering and the Environment, University of Southampton, Southampton SO171BJ, UK

Prof. Dr. V. Subramanya Sarma

Department of Metallurgical and Materials Engineering, Indian Institute of Technology Madras, Chennai 600036, India

\section{[**] Acknowledgements}

This work was supported by the Hungarian Scientific Research Fund, OTKA, Grant no. K109021. Three of the authors were supported by the European Research Council under ERC Grant Agreement No. 267464-SPDMETALS (PHRP, YH and TGL).

\begin{abstract}
The influence of Mo alloying on annealing-induced hardening in ultrafine-grained (UFG) Ni is studied. The hardening observed after low temperature annealing is explained by the annihilation of mobile dislocations and a concomitant clustering of the remaining dislocations into low energy configurations. This study reveals that, with increasing Mo concentration, the hardening effect decreases as the Mo solute atoms hinder the annihilation and rearrangement of dislocations. This trend is the opposite to that observed in electrodeposited Ni-Mo alloys where the larger alloying element concentration yields a higher annealing-induced strengthening effect. The difference is attributed to the different deformation mechanisms in UFG and nanocrystalline Ni-Mo alloys.
\end{abstract}




\section{Introduction}

Bulk ultrafine-grained (UFG) and nanocrystalline materials often exhibit high strength with a concommitant low ductility. ${ }^{[1,2]}$ Former studies showed that short time annealing at moderate temperatures may improve the ductility considerably while the strength decreases only slightly. ${ }^{[3-7]}$ Interestingly, an opposite effect of heat-treatment was also observed in UFG and nanocrystalline solid solutions, namely a hardening caused by annealing. ${ }^{[8-13]}$ In UFG materials processed by severe plastic deformation (SPD) techniques, this effect was explained by the annihilation of mobile dislocations during annealing. ${ }^{[10]}$ Then the reduced mobile dislocation density makes plastic deformation more difficult, leading to hardening. A different mechanism of annealing-induced hardening was observed in nanocrystalline alloys. A recently published study demonstrated that for electrodeposited nanocrystalline NiMo alloys with grain sizes of $\sim 3-25 \mathrm{~nm}$ an annealing at $600-800 \mathrm{~K}$ resulted in hardening. ${ }^{[13]}$ This effect was explained by the segregation of Mo atoms at the grain boundaries which may lead to a more difficult occurrence of grain boundary mediated deformation mechanisms, such as grain boundary sliding. Therefore, for higher Mo concentrations the annealing-induced increase in hardness was found to be larger. In SPD-processed UFG Ni-Mo alloys, the annealing-induced changes in the mechanical performance may differ significantly from the results observed for their electrodeposited nanocrystalline counterparts due to the much larger grain size in the UFG alloys.

In the present work, the evolution of the strength and ductility during annealing of SPDprocessed UFG Ni-Mo alloys was investigated by tension. For this purpose, two alloys with low ( $\sim 0.3$ at.\%) and high ( $\sim$ at.\%) Mo concentrations were processed by high-pressure torsion (HPT) at room temperature (RT). Henceforth, the materials containing $\sim 0.3$ at.\% and $\sim 5$ at.\% Mo are designated Low-Mo and High-Mo, respectively. For a study of the annealinginduced changes in the mechanical behavior, the HPT-processed samples were annealed to the beginning and the end of the exothermic signal observed in a calorimeter. These temperatures 
were 600 and $830 \mathrm{~K}$ for the Low-Mo alloy and 630 and $1000 \mathrm{~K}$ for the High-Mo specimens, respectively. Then, the mechanical performance of the HPT-processed and the annealed specimens was evaluated by tension. The annealing-induced changes in the mechanical performance are correlated to the change of the lattice defect structure (dislocations and grain boundaries). It is noted that only the parameters of the microstructure (e.g., grain size, dislocation density and low-angle grain boundary fraction) are now presented without showing the electron microscopy images and X-ray diffraction patterns which were published previously. ${ }^{[14,15]}$ The same applies for the thermograms determined by calorimetry.

\section{Results and Discussion}

The engineering stress-strain curves for the Low- and High-Mo alloys are shown in Fig. 1a and $\mathbf{b}$, respectively. The yield strength, the ultimate tensile strength, the uniform elongation and the elongation to failure were determined from the tensile curves and plotted for the Lowand High-Mo alloys in Fig. 2a and b, respectively. These values were obtained as the average of the values measured for the two samples fabricated from one disk. The errors in the datum points were calculated as the difference between the average and the individual values obtained for the two tensile specimens. For some datum points, the error is smaller than the symbol size, therefore in this case the uncertainty of the value is represented by the dimension of the symbol. The yield and ultimate tensile strength values for the HPT-processed Low-Mo sample were $\sim 970$ and $\sim 1140 \mathrm{MPa}$, respectively (see Fig. 2a). The higher Mo concentration increased both the yield and ultimate tensile strength to $\sim 1370$ and $\sim 1570 \mathrm{MPa}$, respectively, which can be explained by the higher solute content, the smaller grain size and the higher dislocation density (see Fig. 3).

Annealing of the HPT-processed Low-Mo alloy to $600 \mathrm{~K}$ increased the yield strength and the ultimate tensile strength by 19 and 13\%, respectively (see Fig. 2a). This heat-treatment yielded no change in the ductility as the uniform elongation and the elongation to failure 
remained $\sim 3$ and $\sim 40 \%$, respectively. It should be noted that the present experiments were carried out on miniature tensile specimens for which both the ratio of gauge length to width and the sample thickness are smaller than for a standard tensile specimen. A former study showed that the measured elongation to failure usually increases with decreasing ratio of gauge length to width and increasing specimen thickness. ${ }^{[16]}$ Therefore, the two effects nearly compensate each other by comparing the data obtained on these miniature specimens with a standard sample having dimensions of $5 \times 1 \times 1 \mathrm{~mm}^{3}$. In addition, the present study focuses on the increase of strength due to annealing and not on the change of ductility. For an explanation of the annealing-induced hardening in the Low-Mo sample, the microstructures in the HPT-processed and annealed specimens are compared (see Fig. 3a). During annealing to $\sim 600 \mathrm{~K}$, the dislocation density decreased from $27 \pm 3 \times 10^{14} \mathrm{~m}^{-2}$ to $17 \pm$ $2 \times 10^{14} \mathrm{~m}^{-2}$ while the grain size remained unchanged $(\sim 180 \mathrm{~nm})$ which suggests that only recovery occurred. During this recovery, the dislocation arrangement parameter decreased significantly, indicating a more clustered dislocation configuration after annealing. The more clustered dislocation arrangement within the grains has a higher strengthening effect, resulting in a hardening, despite the decrease of the dislocation density. ${ }^{[17]}$ The arrangement of dislocations into low energy configurations (e.g., into subgrain boundaries) is also confirmed by the increase of the LAGB fraction (see Fig. 3a). The higher strengthening effect of this more clustered dislocation arrangement may cause the hardening observed after annealing to $\sim 600 \mathrm{~K}$, despite the significant reduction in the dislocation density. In addition to this effect, the annihilation of mobile dislocations may also contribute to the hardening observed after annealing since the reduced mobile dislocation density makes plastic deformation more difficult as discussed elsewhere. ${ }^{[10]}$ Moreover, former molecular dynamic simulations showed that the relaxation of non-equilibrium grain boundaries during annealing can lead to a hindered emission of dislocations from grain boundaries which also induces hardening. ${ }^{[8]}$ 
For the High-Mo alloy, annealing to $\sim 630 \mathrm{~K}$ resulted in a much lower hardening than for the Low-Mo alloy (see Fig. 2). During this heat treatment, the dislocation density and the dislocation arrangement parameter decreased while the grain size remained unchanged $(\sim 130$ $\mathrm{nm}$ ), similar to the behavior of the Low-Mo alloy. In addition, the fraction of LAGBs in the High-Mo alloy also increased during annealing to $\sim 630 \mathrm{~K}$. The lower annealing-induced hardening in the High-Mo sample can be explained by the hindering effect of the higher Mo content on the annihilation of mobile dislocations and the clustering of the remaining dislocations. The change of ductility in this specimen was also marginal and similar to the Low-Mo alloy.

An annealing-induced strengthening has already been observed for other UFG or nanocrystalline materials. ${ }^{[8-13]}$ It was shown for electrodeposited nanocrystalline Ni films that the segregation of impurities and alloying elements to the grain boundaries during annealing may lead to a more difficult occurrence of grain boundary mediated deformation mechanisms, such as grain boundary sliding and grain rotation, resulting in hardening. ${ }^{[9,11]}$ As a consequence, the larger alloying element concentration in Ni will yield a higher annealinginduced strengthening effect as was demonstrated for electrodeposited Ni-Mo alloys with grain sizes between $\sim 3$ and $\sim 25 \mathrm{~nm} .{ }^{[13]}$ With increasing Mo content from 0.8 to 21.5 at.\%, the grain size decreased from $\sim 25$ to $\sim 3 \mathrm{~nm}$ and the hardness increment induced by annealing increased from $\sim 20$ to $\sim 125 \%$. At the same time, for the present HPT-processed Ni-Mo alloys the annealing-induced strengthening decreased with increasing Mo content: the increase of the yield strength in the Low-Mo and the High-Mo alloys are $~ 19 \%$ and $~ 2.4 \%$ during annealing to 600 and $630 \mathrm{~K}$, respectively (see Fig. 2). The opposite trend can be explained by the much larger grain size for the HPT-processed Ni-Mo alloys $(\sim 130-180 \mathrm{~nm})$, resulting in a less significant role of grain boundary sliding in plasticity. Rather, for the UFG Ni-Mo alloys the change of the dislocation strengthening during the heat-treatments plays a crucial role in the annealing-induced hardening effect. In the High-Mo alloy, the higher solute Mo content 
hinders the annihilation of mobile dislocations and the arrangement of the remaining dislocations into low energy configurations during the heat-treatment to $\sim 630 \mathrm{~K}$. Therefore, the annealing-induced hardening is less pronounced for the High-Mo with smaller grain size than for the Low-Mo alloy.

For the Low-Mo sample, between 600 and $830 \mathrm{~K}$ the strength values decreased due to the increase of the grain size to $\sim 870 \mathrm{~nm}$ and the reduction of the dislocation density below $\sim 10^{13}$ $\mathrm{m}^{-2}$. In addition, the ductility was significantly improved. The strength reduction and the ductility increase for the High-Mo alloy annealed to the end of the exothermic signal ( 1000 K) were lower than for the Low-Mo sample, despite the larger temperature for the former alloy. This is due to the much better stability of the High-Mo alloy (the gran size increased only to $\sim 300 \mathrm{~nm}$ at $\sim 1000 \mathrm{~K}$ ). It is noted that both alloys remained solid solutions during annealing as proven by X-ray diffraction experiments, therefore there was no influence from precipitation on the mechanical properties.

\section{Conclusions}

1. Annealing to $\sim 600 \mathrm{~K}$ resulted in a considerable hardening for the Low-Mo alloy while the ductility remained unchanged. The annealing-induced hardening was explained by the annihilation of mobile dislocations and the clustering of the remaining dislocations into subgrain boundaries.

2. An increase of the Mo concentration in UFG Ni led to a much lower annealing-induced hardening which was explained by the hindering effect of Mo atoms on the annihilation and clustering of dislocations. Therefore, the influence of Mo content on the annealing-induced hardening in UFG Ni-Mo alloys is opposite to the trend observed formerly for their nanocrystalline counterparts due to the different strengthening mechanisms.

3. Annealing to high temperatures resulted in a simultaneous reduction in the strength and an improvement in the ductility in UFG Ni-Mo alloys. The softening and the increase of ductility 
were lower for the High-Mo alloy, indicating a better thermal stability of this sample compared to the Low-Mo alloy.

\section{Experimental}

$\mathrm{Ni}$ alloys with low ( $\sim 0.3$ at.\%) and high ( $\sim 5$ at. $\%)$ Mo concentrations were used in this study. Disks with diameters of $10 \mathrm{~mm}$ and thicknesses of $1 \mathrm{~mm}$ were processed by HPT at RT for 20 turns under quasi-constrained conditions. ${ }^{[18,19]}$ The applied pressure and the rotation speed were 6.0 GPa and $1 \mathrm{rpm}$, respectively.

During annealing of the HPT-processed Ni-Mo samples in a Perkin Elmer differential scanning calorimeter (DSC2), exothermic peaks were developed in the thermograms (not shown here) due to recovery and recrystallization. For a study of the annealing-induced changes in the mechanical behavior, the HPT-processed samples were annealed to the beginning and the end of the exothermic signal. These temperatures were 600 and $830 \mathrm{~K}$ for the Low-Mo alloy and 630 and $1000 \mathrm{~K}$ for the High-Mo specimen. The higher characteristic temperatures for the High-Mo alloy are due to the better thermal stability. During annealing, the samples were heated to the selected temperatures at a heating rate of $40 \mathrm{~K} / \mathrm{min}$ and then quenched to RT at a rate of $\sim 300 \mathrm{~K} / \mathrm{min}$. The microstructures of the specimens were characterized by electron backscatter diffraction (EBSD) using an FEI Quanta 3D scanning electron microscope (SEM). The EBSD images are not shown here due to space limitations. The average grain sizes and the fraction of the low-angle grain boundaries (LAGBs) were determined from the EBSD data. The grains were considered as the regions in the EBSD images bounded by high-angle grain boundaries (HAGBs) having misorientation angle $>15^{\circ}$. The number-averaged grain size values were then determined for the HPT-processed and the annealed samples. The LAGBs were defined as boundaries with misorientations between 2 and $15^{\circ}$. 
The dislocations in the SPD-processed and the annealed Ni alloys were characterized by Xray line profile analysis (XLPA). The diffraction patterns were measured by a rotating anode $\mathrm{X}$-ray diffractometer (manufacturer: Rigaku) using $\mathrm{CuK} \alpha_{1}$ radiation. The average dislocation density and the dislocation arrangement parameter were determined by the CMWP fitting procedure. ${ }^{[20]}$ The dislocation arrangement parameter characterizes the clustering of dislocations. Smaller values of the dislocation arrangement parameter denote a more shielded strain field of dislocations due to their arrangement into low energy configurations, such as low-angle grain boundaries or dipolar walls.

Tensile specimens were prepared from the disks processed by HPT and the samples annealed after HPT. Two miniature tensile specimens were cut from each HPT-processed disk at the half-radius by electro-discharge machining (EDM). These two miniature tensile specimens were cut symmetrically on either side of the centre of the disk. The thickness of the HPTprocessed disk was $\sim 0.80 \mathrm{~mm}$. However, as a consequence of the elastic distortion of the anvils during HPT, the surface of the HPT-processed disk became curved. ${ }^{[21]}$ So the upper and lower surfaces of the HPT-processed disks were ground using abrasive papers to a thickness of $\sim 0.60 \mathrm{~mm}$ before EDM cutting in order to obtain a uniform thickness and remove any surface irregularities. The gauge length and width of the tensile specimens were 1.1 and 0.95 mm, respectively. Tensile tests were preformed on a Zwick Z030 testing machine. All miniature tensile specimens were pulled to failure at room temperature with an initial strain rate of $10^{-3} \mathrm{~s}^{-1}$.

Keywords: Ni-Mo alloys; ultrafine-grained microstructure; dislocations; annealing; hardening

Received: ((will be filled in by the editorial staff))

Revised: ((will be filled in by the editorial staff)) Published online: ((will be filled in by the editorial staff)) 
[1] R.Z. Valiev, A.P. Zhilyaev, T.G. Langdon, Bulk Nanostructured Materials: Fundamentals and Applications, John Wiley \& Sons, Inc., Hoboken, NJ, 2014.

[2] N. Tsuji, Y. Ito, Y. Saito, Y. Minamino, Scripta Mater. 2002, 47, 893.

[3] Y.M. Wang, E. Ma, Acta Mater. 2004, 52, 1699.

[4] Y. Wang, M. Chen, F. Zhou, E. Ma, Nature 2002, 419, 912.

[5] Y.H. Zhao, T. Topping, J.F. Bingert, J.J. Thornton, A.M. Dangelewicz, Y. Li, W. Liu, Y.T. Zhu, Y.Z. Zhou, E.J. Lavernia, Advanced Mater. 2008, 20, 3028.

[6] K. Sitarama Raju, V. Subramanya Sarma, A. Kauffmann, Z. Hegedűs, J. Gubicza, M. Peterlechner, J. Freudenberger, G. Wilde, Acta Mater. 2013, 61, 228.

[7] O. Andreau, J. Gubicza, N.X. Zhang, Y. Huang, P. Jenei, T.G. Langdon, Mater. Sci. Eng. A 2014, 615, 231.

[8] A. Hasnaoui, H. Van Swygenhoven, P.M. Derlet, Acta Mater. 2002, 50, 3927.

[9] Y.M. Wang, S. Cheng, Q.M. Wei, E. Ma, T.G. Nieh, A. Hamza, Scripta Mater. 2004, 51, 1023.

[10] X. Huang, N. Hansen, N. Tsuji, Science 2006, 312, 249.

[11] L. Chang, P.W. Kao, C.-H. Chen, Scripta Mater. 2007, 56, 713.

[12] J. Tao, G. Chen, W. Jian, J. Wang, Y. Zhu, X. Zhu, T.G. Langdon, Mater. Sci. Eng. A 2015, 628, 207.

[13] J. Hu, Y. N. Shi, X. Sauvage, G. Sha, K. Lu, Science 2017, 355, 1292.

[14] G. Kapoor, Y. Huang, V. Subramanya Sarma, T.G. Langdon, J. Gubicza, Mater. Sci. Eng. A 2017, 688, 92.

[15] G. Kapoor, Y. Huang, V. Subramanya Sarma, T.G. Langdon, J. Gubicza, Mater. Char. 2017, 130, 56 .

[16] Y.H. Zhao, Y.Z. Guo, Q. Wei, A.M. Dangelewicz, C. Xu, Y.T. Zhu, T.G. Langdon, Y.Z. Zhou, E.J. Lavernia, Scripta Mater. 2008, 59, 627.

[17] F. Hernandez Olivares, J. Gil Sevillano, Acta Metall. 1987, 35, 631. 
[18] A.P. Zhilyaev, T.G. Langdon, Prog. Mater. Sci. 2008, 53, 893.

[19] R.B. Figueiredo, P.R. Cetlin, T.G. Langdon, Mater. Sci. Eng. A 2011, 528, 8198.

[20] G. Ribárik, J. Gubicza, T. Ungár, Mater. Sci. Eng. A 2004, 387-389, 343.

[21] P.H.R. Pereira, R.B. Figueiredo, P.R. Cetlin, T.G. Langdon, Mater. Sci. Eng. A 2015, 631,201

a)

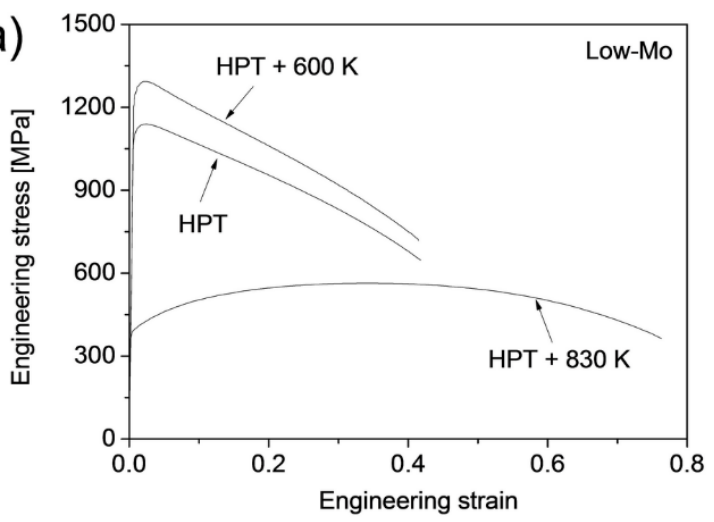

b)

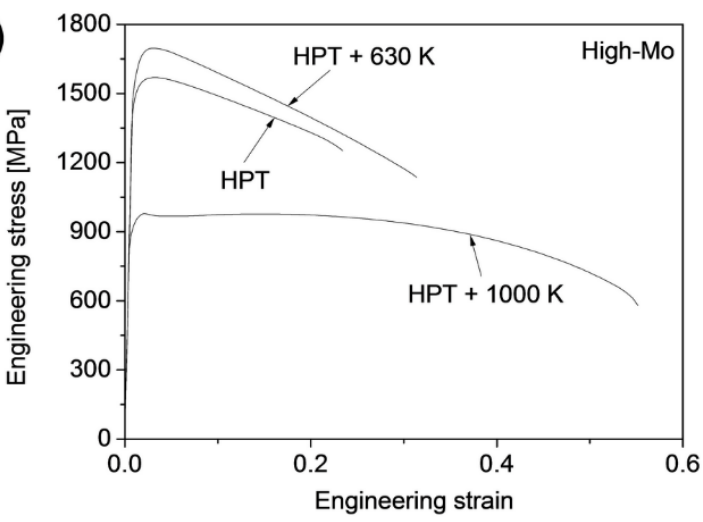

Figure 1: Engineering stress-strain curves obtained by tension for the HPT-processed and annealed states of (a) the Low- and (b) High-Mo alloys.
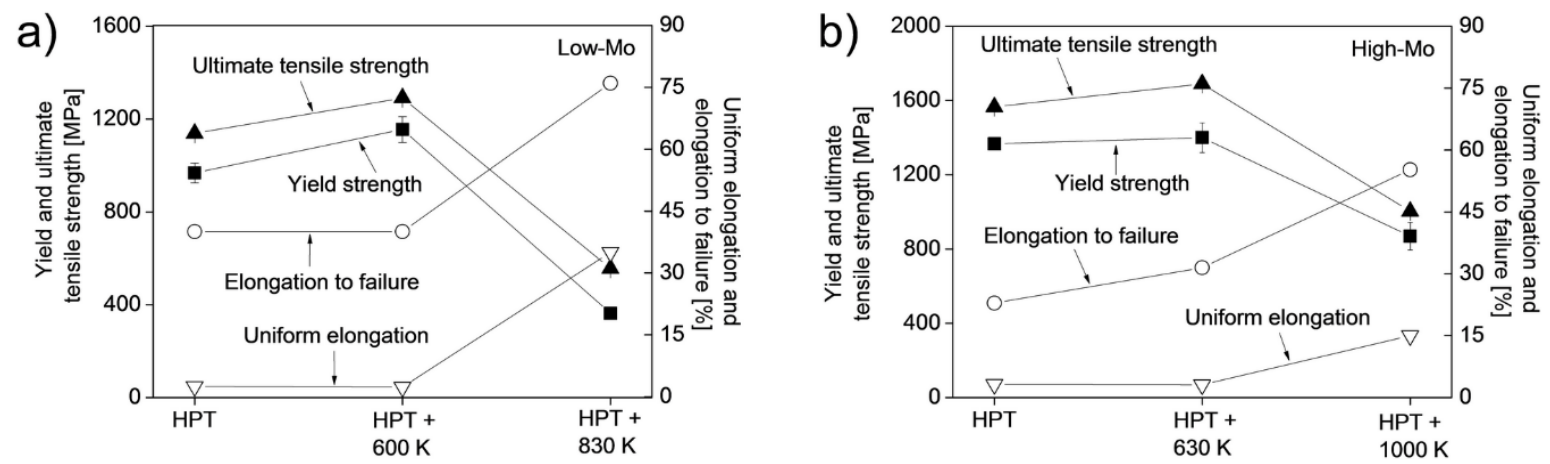

Figure 2: The yield strength, the ultimate tensile strength, the uniform elongation and the elongation to failure determined for the HPT-processed and annealed states of (a) the Lowand (b) High-Mo alloys. 

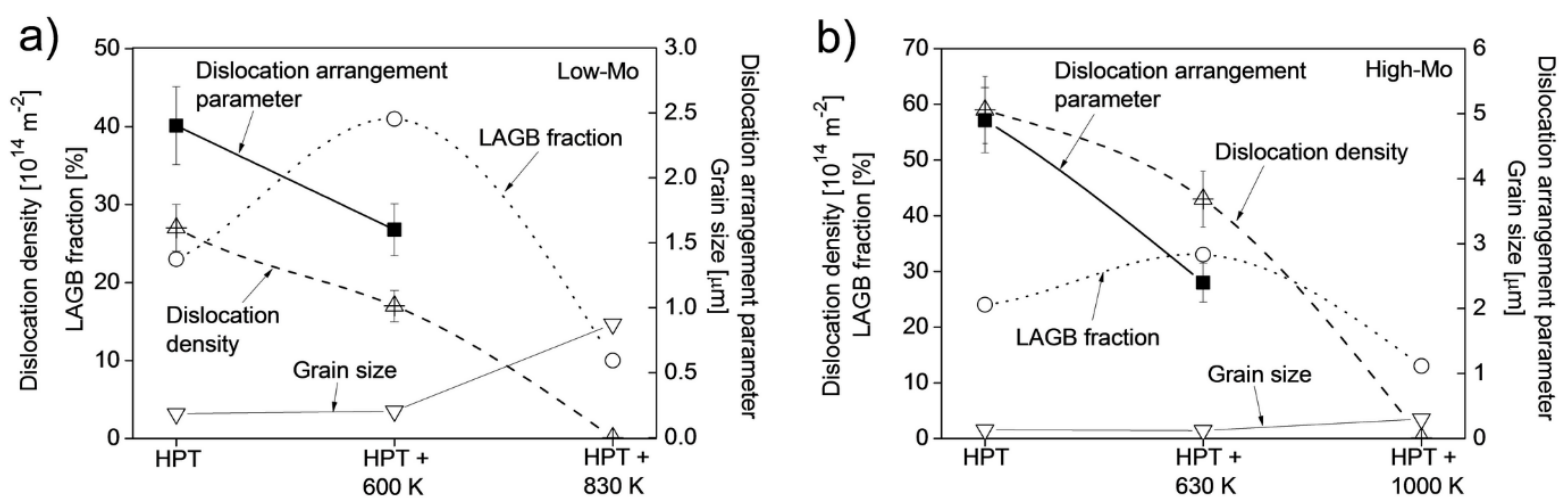

Figure 3: The grain size, the dislocation density, the dislocation arrangement parameter and the LAGB fraction determined for the HPT-processed and annealed states of (a) the Low-and (b) High-Mo alloys. 
An annealing-induced hardening is observed in severely deformed ultrafine-grained Ni-Mo alloys. This effect is explained by the annihilation of mobile dislocations and a concomitant clustering of the remaining dislocations into low energy configurations. With increasing Mo concentration in ultrafine-grained $\mathrm{Ni}$ alloys, this hardening effect decreases as Mo solute atoms hinder the annihilation and rearrangement of dislocations.

Jenö Gubicza, Pedro Henrique R. Pereira, Garima Kapoor, Yi Huang, V. Subramanya Sarma, and Terence G. Langdon

Annealing-induced Hardening in Ultrafine-grained Ni-Mo Alloys

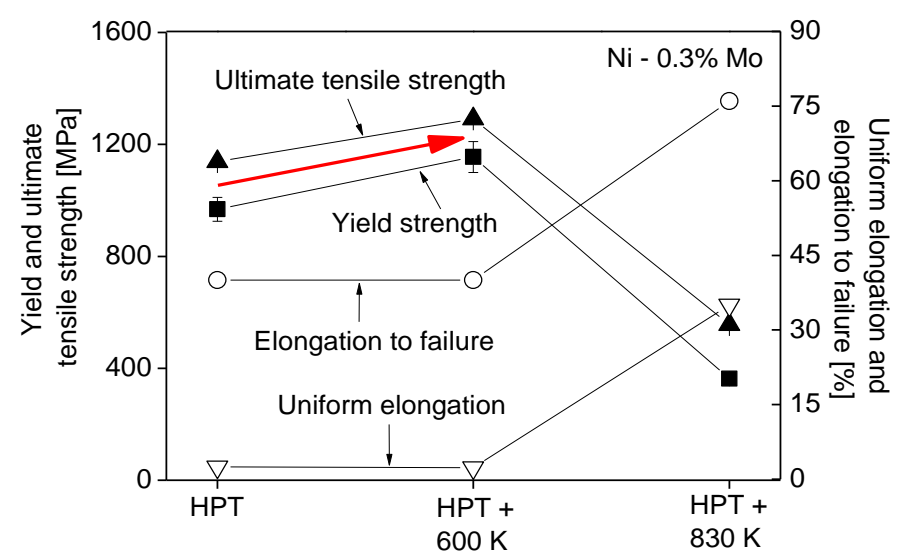

